\title{
Component-wise positivity of solutions to periodic boundary problem for linear functional differential system
}

\author{
Alexander Domoshnitsky ${ }^{1 *}$, Robert Hakl ${ }^{2}$ and Jiř́ Šremr ${ }^{2}$
}

\footnotetext{
* Correspondence: adom@ariel.ac.il ${ }^{1}$ Department of Mathematics and Computer Science, The Ariel University Center of Samaria, 44837 Ariel Israel

Full list of author information is available at the end of the article
}

\begin{abstract}
The classical Ważewski theorem claims that the condition $p_{i j} \leq 0, j \neq i, i, j=1, \ldots, n$, is necessary and sufficient for non-negativity of all the components of solution vector to a system of the inequalities $x^{\prime}(t)+\sum_{j=1}^{n} p_{i j}(t) x(t) \geq 0, x_{i}(0) \geq 0, i=1, \ldots, n$. Although this result was extent on various boundary value problems and on delay differential systems, analogs of these heavy restrictions on non-diagonal coefficients $p_{i j}$ preserve in all assertions of this sort. It is clear from formulas of the integral representation of the general solution that these theorems claim actually the positivity of all elements of Green's matrix. The method to compare only one component of the solution vector, which does not require such heavy restrictions, is proposed in this article. Note that comparison of only one component of the solution vector means the positivity of elements in a corresponding row of Green's matrix. Necessary and sufficient conditions of this fact are obtained in the form of theorems about differential inequalities. It is demonstrated that the sufficient conditions of positivity of the elements in the nth row of Green's matrix, proposed in this article, cannot be improved in corresponding cases. The main idea of our approach is to construct a first order functional differential equation for the $n$th component of the solution vector and then to use assertions, obtained recently for first order scalar functional differential equations. This demonstrates the importance to study scalar equations written in a general operator form, where only properties of the operators and not their forms are assumed. Note that in some cases the sufficient conditions, obtained in the article, does not require any smallness of the interval $[0, \omega]$, where the system is considered.
\end{abstract}

Mathematics Subject Classification 2000: 34K06; 34K10.

Keywords: periodic problem, Green's matrix, linear functional differential system, positive solution, negative solution

\section{Introduction}

Consider the following system of functional differential equations

$$
\left(M_{i} x\right)(t) \equiv x_{i}^{\prime}(t)+\sum_{j=1}^{n}\left(B_{i j} x_{j}\right)(t)=f_{i}(t), \quad t \in[0, \omega], \quad i=1, \ldots, n,
$$

\section{Springer}


subjected to the periodic conditions

$$
x_{i}(0)=x_{i}(\omega)+c_{i}, \quad i=1, \ldots, n,
$$

where $x=\operatorname{col}\left(x_{1}, \ldots, x_{n}\right), B_{i j}: C_{[0, \omega]} \rightarrow L_{[0, \omega]}, i, j=1, \ldots, n$, are linear bounded operators, $C_{[0, \omega]}$ and $L_{[0, \omega]}$ are the spaces of continuous and summable functions $y:[0, \omega] \rightarrow R^{1}$, respectively, $f_{i} \in L_{[0, \omega]}, c_{i} \in R^{1}, i=1, \ldots, n$.

The operators $B_{i j}$ can be, for example, of the following forms:

$$
\begin{aligned}
& \left(B_{i j} x\right)(t)=\sum_{k=1}^{m} p_{i j}^{k}(t) x\left(h_{k}(t)\right), t \in[0, \omega], \\
& \left(B_{i j} x\right)(t)=\int_{0}^{\omega} K_{i j}(t, s) x(s) d s, t \in[0, \omega],
\end{aligned}
$$

and also their linear combinations or superpositions are allowed, where $p_{i j}^{k}:[0, \omega] \rightarrow R^{1}, h_{k}:[0, \omega] \rightarrow[0, \omega]$, and $K_{i j}:[0, \omega] \times[0, \omega] \rightarrow R^{1}$ are suitable functions.

If the homogeneous periodic problem

$$
\begin{aligned}
& \left(M_{i} x\right)(t)=0, \quad t \in[0, \omega], \quad i=1, \ldots, n, \\
& x_{i}(0)=x_{i}(\omega), \quad i=1, \ldots, n,
\end{aligned}
$$

has only the trivial solution, then the boundary value problem (1.1), (1.2) has for each $f=\operatorname{col}\left(f_{1}, \ldots, f_{n}\right)$ with $f_{i} \in L_{[0, \omega]}$ and $c=\operatorname{col}\left(c_{1}, \ldots, c_{n}\right)$ with $c_{i} \in R^{1}, i=1, \ldots, n$, a unique solution $x$, which has the following representation [1] (see also [2,3])

$$
x(t)=\int_{0}^{\omega} G(t, s) f(s) d s+X(t) c, \quad t \in[0, \omega],
$$

where the $n \times n$ matrix $G(t, s)$ is called Green's matrix of periodic problem (1.1), (1.4) and the $n \times n$ matrix $X(t)$ is the fundamental matrix of the system (1.3) such that $X(0)-X(\omega)=E$, where $E$ is the unit $n \times n$ matrix. It is clear from the solution representation (1.5) that the matrices $G(t, s)$ and $X(t)$ determine all properties of solutions.

The following property is the basis of the approximate integration method by Tchaplygin [4]: from the conditions

$$
\left(M_{i} x\right)(t) \geq\left(M_{i} y\right)(t), \quad t \in[0, \omega], \quad i=1, \ldots, n, \quad l x \geq l y,
$$

where $l: C_{[0, \omega]}^{n} \rightarrow R^{n}$ is a linear bounded functional, it follows that

$$
x_{i}(t) \geq y_{i}(t), \quad t \in[0, \omega], \quad i=1, \ldots, n .
$$

Series of articles, started with the known article by Luzin [5], were devoted to the various aspects of Tchaplygin's approximate method. The monograph by Lakshmikantham and Leela [6] was one of important in this area. In the book by Krasnosel'skii et al. [7], which was devoted to approximate methods for operator equations, property $(1.6) \Rightarrow(1.7)$ was also essentially used. These ideas have been developing in scores of books on the monotone technique for approximate solution of boundary value problems for systems of differential equations. Note in this connection the works by 
Kiguradze and Půža $[3,8,9]$. From formula of solution representation (1.5) it is clear that property $(1.6) \Rightarrow(1.7)$ with $l x \equiv x(0)-x(\omega)$ is true if and only if all elements of the matrices $G(t, s)$ and $X(t)$ are non-negative.

As a particular case of system (1.1) let us consider the following system

$$
x_{i}^{\prime}(t)+\sum_{j=1}^{n} p_{i j}(t) x_{j}\left(h_{i j}(t)\right)=f_{i}(t), \quad t \in[0, \omega], \quad i=1, \ldots, n,
$$

where $p_{i j} \in L_{[0, \omega]}$ and $h_{i j}:[0, \omega] \rightarrow[0, \omega]$ are measurable functions. The classical Ważewski's [10] theorem claims that the condition

$$
p_{i j}(t) \leq 0, t \in[0, \omega], \quad i, j=1, \ldots, n, \quad i \neq j,
$$

is necessary and sufficient for the property $(1.6) \Rightarrow(1.7)$ with $l x \equiv x(0)$ for system of ordinary differential equations

$$
x_{i}^{\prime}(t)+\sum_{j=1}^{n} p_{i j}(t) x_{j}(t)=f_{i}(t), \quad t \in[0, \omega], \quad i=1, \ldots, n .
$$

Extensions of this Ważewski's theorem on various boundary value problems with functional differential equations and boundary conditions were obtained in [3] which defines the modern level in this topic. But all these results are based on analogs of condition (1.9), which, of course, are close to necessary conditions for the property $(1.6) \Rightarrow(1.7)$.

In many problems in practice one needs to compare only one component of solution vector. Let us change the formulation and focus our attention upon the problem of comparison for only one of the components of solution vector. Let $k_{i}$ be either 1 or 2 . In this article we consider the following property: when from the conditions

$$
(-1)^{k_{i}}\left[\left(M_{i} x\right)(t)-\left(M_{i} y\right)(t)\right] \geq 0, \quad t \in[0, \omega], \quad i=1, \ldots, n, \quad l x=l y
$$

it does follow that, for a fixed $r \in\{1, \ldots, n\}$, the components $x_{r}$ and $y_{r}$ of vectors $x$ and $y$ satisfy the inequality

$$
x_{r}(t) \geq y_{r}(t), \quad t \in[0, \omega] .
$$

This property is a weakening of the property $(1.6) \Rightarrow(1.7)$ and, as we will obtain below, leads to essentially less hard limitations on the given system. From formula of solution's representation (1.5) it follows that this property is reduced to sign-constancy of all elements standing only in the $r$ th row of Green's matrix.

The article is built as follows. Auxiliary results on positivity (negativity) of Green's matrices in the case when all nondiagonal operators $B_{i j}(i \neq j, i, j=1, \ldots, n)$ are negative (positive) are obtained in Section 2. In Theorem 2.1 the results of this sort are presented as three equivalent facts: the first of them is an assertion about differential inequality, the second-about positivity (negativity) of elements of Green's matrix, and the third-about the spectral radius of a corresponding auxiliary operator. In Theorem 2.2 we choose a constant vector as a test vector function, that simplifies the assertion about differential inequalities and allows us to obtain then estimates of elements of Green's matrix. 
The main results of this article about positivity or negativity of elements in the nth row of Green's matrix are formulated in Section 3. General assertions are presented in Theorems 3.1 and 3.2. In Corollary 3.1 we avoid assumptions about positivity (negativity) of Green's functions of auxiliary equations and formulate all assumptions in the form of simple algebraic inequalities.

The proofs of formulated assertions of Sections 2 and 3 are presented in Section 4 . The main idea of our approach is to construct a corresponding scalar functional differential equation of the first order

$$
x_{n}^{\prime}(t)+\left(B x_{n}\right)(t)=f^{*}(t), \quad t \in[0, \omega],
$$

for $n$th component of a solution vector, where $B: C_{[0, \omega]} \rightarrow L_{[0, \omega]}$ is a linear continuous operator, $f^{\prime \prime} \in L_{[0, \omega]}$. This equation is built in Section $4[11,12]$. Then the technique of analysis of the first order scalar functional differential equations, developed, for example, in the works [13,14], is used. On this basis we prove assertions of Section 3.

The results about positivity and negativity of elements of Green's matrices of second order systems with deviating arguments are presented in Section 5.

Note that results of this sort for the Cauchy problem (i.e., if $l x \equiv x(0)$ ) and Volterra (according to Tikhonov's definition) operators $B_{i j}: C_{[0, \omega]} \rightarrow L_{[0, \omega]}$ were proposed in the recent article [11], where the obtained operator $B: C_{[0, \omega]} \rightarrow L_{[0, \omega]}$ became a Volterra operator. In this article we consider the periodic problem that implies that the operator $B: C_{[0, \omega]} \rightarrow L_{[0, \omega]}$ is not a Volterra one even in the case when all $B_{i j}: C_{[0, \omega]} \rightarrow L_{[0, \omega]}, i$, $j=1, \ldots, n$, are Volterra operators.

For our purpose we recall that the Tikhonov's definition can be generalised in the following manner: An operator $B: C_{[0, \omega]} \rightarrow L_{[0, \omega]}$ is called 0-Volterra, respectively $\omega$ Volterra, if $(B x)(t)=0$ for $t \in[0, c]$, respectively for $t \in[c, \omega]$, whenever $c \in[0, \omega]$ and $x(t)=0$ for $t \in[0, c]$, respectively for $t \in[c, \omega]$.

\section{Positivity of Green's matrices of systems of FDEs-auxiliary assertions}

In this section we consider the inhomogeneous system (1.1) with the homogeneous boundary conditions (1.4), where as above the operators $B_{i j}: C_{[0, \omega]} \rightarrow L_{[0, \omega]}$ are supposed to be linear and bounded.

Assuming that, for any $i=1, \ldots, n$, the scalar periodic problem

$$
\begin{aligned}
& x_{i}^{\prime}(t)+\left(B_{i i} x_{i}\right)(t)=f_{i}(t), t \in[0, \omega], \\
& x_{i}(0)=x_{i}(\omega),
\end{aligned}
$$

is uniquely solvable for every $f_{i} \in L_{[0, \omega]}$, we define the operator $R: C_{[0, \omega]}^{n} \rightarrow C_{[0, \omega]}^{n}$ acting in the space of $n$-dimensional vector functions with continuous elements $x_{i}$ : [0, $\omega] \rightarrow R^{1}$ endowed with the norm $\|x\|_{C_{[0, \omega]}^{n}}=\max _{1 \leq i \leq n t \in[0, \omega]}\left|x_{i}(t)\right|$ as follows:

$$
(R x)(t)=\operatorname{col}\left(-\int_{0}^{\omega} g_{i}(t, s) \sum_{j=1, j \neq i}^{n}\left(B_{i j} x_{j}\right)(s) d s\right)_{i=1}^{n}, \quad t \in[0, \omega],
$$

where $g_{i}(t, s)$ denotes Green's function of the problem (2.1), (2.2).

Note that properties of Green's functions are studied in the book [1], where foundations of the general theory of functional differential equations are established. It was obtained 
that $g_{i}(\cdot, s)$ is absolutely continuous for almost every (a.e.) $s \in[0, \omega]$ on each of the intervals $[0, s)$ and $(s, \omega]$. Below, talking, for example, about positivity of Green's function in the rectangle $t, s \in[0, \omega]$, we actually mean $g_{i}(t, s)>0$ for $t \in[0, \omega]$ and a.e. $s \in[0, \omega]$. Analogously, if we say that Green's functions $g_{i}(t, s)$ for $1=1, \ldots, n$ preserve their signs, we mean that for every $i$ either $g_{i}(t, s) \geq 0$ or $g_{i}(t, s) \leq 0$ for $t \in[0, \omega]$ and a.e. $s \in[0, \omega]$.

The following assertion follows from Theorem 3.1 of the article [11], similar results can be found also in the monograph [3].

Theorem 2.1. Let the following conditions be fulfilled:

(1) Green's functions $g_{i}(t, s), i=1, \ldots, n$, of $n$ scalar periodic problems (2.1), (2.2) exist, preserve their signs and are such that

$$
\int_{0}^{\omega}\left|g_{i}(t, s)\right| \varphi(s) d s>0, \quad t \in[0, \omega]
$$

for each positive measurable essentially bounded function $\phi$,

(2) the non-diagonal operators $B_{i j}, i, j=1, \ldots, n, i \neq j$, are positive or negative such that the operator $R: C_{[0, \omega]}^{n} \rightarrow C_{[0, \omega]}^{n}$ determined by the formula (2.3) is positive.

Then the following assertions are equivalent:

(a) there exists a vector function $v \in C_{[0, \omega]}^{n}$ with positive absolutely continuous components $v_{i}:[0, \omega] \rightarrow(0,+\infty)$ such that

$$
\int_{0}^{\omega} g_{i}(t, s)\left(M_{i} v\right)(s) d s>0, \quad t \in[0, \omega], \quad i=1, \ldots, n,
$$

(b) boundary value problem (1.1) and (1.4) is uniquely solvable for each right hand side $f=\operatorname{col}\left(f_{1}, \ldots, f_{n}\right)$ such that $f_{i} \in L_{[0, \omega]}, i=1, \ldots, n$, and the elements $G_{i j}(t, s), i, j=1$, ..., $n$, of its Green's matrix $G(t, s)$ preserve their signs and satisfy the inequality

$$
g_{i}(t, s) G_{i j}(t, s) \geq 0, \quad t, s \in[0, \omega]
$$

while

$$
\left|G_{i i}(t, s)\right| \geq\left|g_{i}(t, s)\right|, \quad t, s \in[0, \omega],
$$

for $i, j=1, \ldots, n$,

(c) the spectral radius of the operator $R: C_{[0, \omega]}^{n} \rightarrow C_{[0, \omega]}^{n}$ is less than one.

The following assertion follows from Theorem 2.1 if we set $v(t) \equiv \operatorname{col}\left(z_{1}, \ldots, z_{n}\right)$ as a constant vector with positive components.

Theorem 2.2. Let the following conditions be fulfilled:

(1) Green's functions $g_{i}(t, s), i=1, \ldots, n$, of $n$ scalar periodic problems (2.1), (2.2) exist, are non-negative (respectively, non-positive), and satisfy inequality (2.4) for each positive measurable essentially bounded function $\phi$,

(2) all non-diagonal operators $B_{i j}, i, j=1, \ldots, n, i \neq j$, are negative (respectively, positive),

(3) there exists a vector $z=\operatorname{col}\left(z_{1}, \ldots, z_{n}\right)$ with all positive components such that

$$
\sum_{j=1}^{n}\left(B_{i j} z_{j}\right)(t) n \geq 1, \quad t \in[0, \omega], \quad i=1, \ldots, n,
$$


respectively,

$$
\sum_{j=1}^{n}\left(B_{i j} z_{j}\right)(t) n \leq-1, \quad t \in[0, \omega], \quad i=1, \ldots, n .
$$

Then boundary value problem (1.1), (1.4) is uniquely solvable for each right hand side $f=\operatorname{col}\left(f_{1}, \ldots, f_{n}\right)$, all elements $G_{i j}(t, s), i, j=1, \ldots, n$, of its Green's matrix $G(t, s)$ are nonnegative (respectively, non-positive) and the following inequalities

$$
\int_{0}^{\omega} \sum_{j=1}^{n}\left|G_{i j}(t, s)\right| d s \leq z_{i}, \quad t \in[0, \omega], \quad i=1, \ldots, n .
$$

hold.

Remark 2.1. It is clear that assumption (3) in Theorem 2.2 is fulfilled if and only if there exists a vector $\bar{z}=\operatorname{col}\left(\bar{z}_{1}, \ldots, \bar{z}_{n}\right)$ with all positive components and $\varepsilon>0$ such that

$$
\sum_{j=1}^{n}\left(B_{i j} \bar{z}_{j}\right)(t) \geq \varepsilon, \quad t \in[0, \omega], \quad i=1, \ldots, n,
$$

respectively,

$$
\sum_{j=1}^{n}\left(B_{i j} \bar{z}_{j}\right)(t) \leq-\varepsilon, \quad t \in[0, \omega], \quad i=1, \ldots, n .
$$

For system with argument deviations (1.8), the condition (2.10) looks as the following one

$$
\sum_{j=1}^{n} p_{i j}(t) \bar{z}_{j} \geq \varepsilon, \quad t \in[0, \omega], \quad i=1, \ldots, n
$$

that coincides with the fact that $P(t)=\left\{p_{i j}(t)\right\}_{i, j=1}^{n}$ is $M$-matrix (see [15]).

If we set $\bar{z}_{j}=1$ for $j=1, \ldots, n$, then we get

$$
\sum_{j=1}^{n} p_{i j}(t) \geq \varepsilon, \quad t \in[0, \omega], \quad i=1, \ldots, n,
$$

which can be interpreted as the main diagonal dominance and condition (2.11) as its generalization.

Remark 2.2. If instead of assumption (3) in Theorem 2.2 we assume that there exists a constant matrix $Y=\left\{y_{i j}\right\}_{i, j=1}^{n}$ with non-negative components possessing the property

$$
\sum_{j=1}^{n} y_{i j}>0, \quad i=1, \ldots, n,
$$

such that

$$
\sum_{k=1}^{n}\left(B_{i k} y_{k j}\right)(t) \geq \delta_{i j,} \quad t \in[0, \omega], \quad i, j=1, \ldots, n,
$$


respectively,

$$
\sum_{k=1}^{n}\left(B_{i k} y_{k j}\right)(t) \leq-\delta_{i j}, \quad t \in[0, \omega], \quad i, j=1, \ldots, n,
$$

where

$$
\delta_{i j}= \begin{cases}1 & \text { if } i=j, \\ 0 & \text { if } i \neq j,\end{cases}
$$

then the elements $G_{i j}(t, s), i, j=1, \ldots, n$, of the Green's matrix $G(t, s)$ satisfy the inequalities

$$
\int_{0}^{\omega}\left|G_{i j}(t, s)\right| d s \leq y_{i j}, \quad t \in[0, \omega], \quad i, j=1, \ldots, n .
$$

Notation 2.1. Let $B: C_{[0, \omega]} \rightarrow L_{[0, \omega]}$ be a linear operator. For any $t \in[0, \omega]$ define the sets

$$
H_{*}(t)=\left\{z \in[0, \omega]: x \in C_{[0, \omega]}, \quad x(s)=0, s \in[z, \omega] \Rightarrow(B x)(t)=0\right\}
$$

and

$$
H^{*}(t)=\left\{z \in[0, \omega]: x \in C_{[0, \omega]}, \quad x(s)=0, s \in[0, z] \Rightarrow(B x)(t)=0\right\} .
$$

Note that it can be shown that the sets $H^{*}(t)$ and $H^{*}(t)$ are nonempty for almost every $t \in[0, \omega]$ and thus we can define

$$
h_{*}(t)=\sup H_{*}(t) \quad \text { for almost every } t \in[0, \omega]
$$

and

$$
h^{*}(t)=\inf H^{*}(t) \text { for almost every } t \in[0, \omega] .
$$

Corollary 2.1. Let the following conditions be fulfilled:

(1) the operators $B_{i i}, i=1, \ldots, n$, admit the representation $B_{i i}=B_{i i}^{+}-B_{i i}^{-}$, where $B_{i i}^{+}, B_{i i}^{-}: C_{[0, \omega]} \rightarrow L_{[0, \omega]}$ are linear positive operators, and at least one of the following assumptions (1a) or (1b) is satisfied:

(a) $B_{i i}^{+} B_{i i}^{-}, i=1, \ldots, n$, are 0 -Volterra operators,

$$
\left(B_{i i} 1\right)(t) \geq 0, \quad t \in[0, \omega], \quad B_{i i} 1 \not \equiv 0, \quad i=1, \ldots, n
$$

and

$$
\int_{h_{i i *}^{+}(t)}^{t}\left(B_{i i}^{+} 1\right)(s) d s \leq \frac{1}{e}, \quad t \in[0, \omega], \quad i=1, \ldots, n,
$$

(b) the inequalities

$$
\left\|B_{i i}^{+} 1\right\|_{L}<1, \quad\left\|B_{i i}^{-} 1\right\|_{L}<1,
$$

and

$$
\frac{\left\|B_{i i}^{-} 1\right\|_{L}}{1-\left\|B_{i i}^{-} 1\right\|_{L}}<\left\|B_{i i}^{+} 1\right\|_{L}
$$


hold for $i=1, \ldots, n$,

(2) all non-diagonal operators $B_{i j}, i, j=1, \ldots, n, i \neq j$, are negarive,

(3) there exists a vector $z=\operatorname{col}\left(z_{1}, \ldots, z_{n}\right)$ with all positive components such that (2.7) holds.

Then boundary value problem (1.1), (1.4) is uniquely solvable for each right hand side $f=\operatorname{col}\left(f_{1}, \ldots, f_{n}\right)$, all elements $G_{i j}(t, s), i, j=1, \ldots, n$, of its Green's matrix $G(t, s)$ are nonnegative and

$$
\int_{0}^{\omega} \sum_{j=1}^{n} G_{i j}(t, s) d s \leq z_{i}, \quad t \in[0, \omega], \quad i=1, \ldots, n .
$$

The proof is based on Theorem 2.2 and Lemmas 4.2 and 4.4 from the next section.

Remark 2.3. If instead of assumption (3) in Corollary 2.1 we assume that there exists a constant matrix $Y=\left\{y_{i j}\right\}_{i, j=1}^{n}$ with non-negative components possessing the property (2.12) such that relations (2.13) are fulfilled with $\delta_{i j}$ given by (2.15), then the elements $G_{i j}(t, s), i, j=1, \ldots, \mathrm{n}$, of the Green's matrix $G(t, s)$ satisfy the inequalities

$$
\int_{0}^{\omega} G_{i j}(t, s) d s \leq y_{i j}, \quad t \in[0, \omega], \quad i, j=1, \ldots, n .
$$

Corollary 2.2. Let the following conditions be fulfilled:

(1) the operators $B_{i i}, i=1, \ldots, n$, admit the representation $B_{i i}=B_{i i}^{+}-B_{i i}^{-}$, where $B_{i i}^{+}, B_{i i}^{-}: C_{[0, \omega]} \rightarrow L_{[0, \omega]}$, are linear positive operators, and at least one of the following assumptions (1a) or (1b) is satisfied:

(a) $B_{i i}^{+}, B_{i i}^{-}, i=1, \ldots, n$, are $\omega$-Volterra operators,

$$
\left(B_{i i} 1\right)(t) \leq 0, \quad t \in[0, \omega], \quad B_{i i} 1 \not \equiv 0, \quad i=1, \ldots, n
$$

and

$$
\int_{t}^{h_{i i}^{-*}(t)}\left(B_{i i}^{-} 1\right)(s) d s \leq \frac{1}{e}, \quad t \in[0, \omega], \quad i=1, \ldots, n,
$$

(b) the inequalities (2.18) and

$$
\frac{\left\|B_{i i}^{+} 1\right\|_{L}}{1-\left\|B_{i i}^{+} 1\right\|_{L}}<\left\|B_{i i}^{-} 1\right\|_{L}
$$

hold for $i=1, \ldots, n$,

(2) all non-diagonal operators $B_{i j}, i, j=1, \ldots, n, i \neq j$, are positive,

(3) there exists a vector $z=\operatorname{col}\left(z_{1}, \ldots, z_{n}\right)$ with all positive components such that (2.8) holds.

Then boundary value problem (1.1), (1.4) is uniquely solvable for each right hand side $f=\operatorname{col}\left(f_{1}, \ldots, f_{n}\right)$, all elements $G_{i j}(t, s), i, j=1, \ldots, n$, of its Green's matrix $G(t, s)$ are nonpositive and

$$
-\int_{0}^{\omega} \sum_{j=1}^{n} G_{i j}(t, s) d s \leq z_{i}, \quad t \in[0, \omega], \quad i=1, \ldots, n .
$$


The proof is based on Theorem 2.2 and Lemmas 4.3 and 4.5 from the next section.

Remark 2.4. If instead of assumption (3) in Corollary 2.2 we assume that there exists a constant matrix $Y=\left\{y_{i j}\right\}_{i, j=1}^{n}$ with non-negative components possessing the property (2.12) such that relations (2.14) are fulfilled with $\delta_{i j}$ given by (2.15), then the elements $G_{i j}(t, s), i, j=1, \ldots, n$, of the Green's matrix $G(t, s)$ satisfy the inequalities

$$
-\int_{0}^{\omega} G_{i j}(t, s) d s \leq y_{i j}, \quad t \in[0, \omega], \quad i, j=1, \ldots, n .
$$

Consider now the particular case of system (1.1)

$$
x_{i}^{\prime}(t)+\sum_{k=1}^{m} \sum_{j=1}^{n} p_{i j}^{k}(t) x_{j}\left(h_{i j}^{k}(t)\right)=f_{i}(t), \quad t \in[0, \omega], \quad i=1, \ldots, n,
$$

where $p_{i j^{\prime}}^{k} f_{i}:[0, \omega] \rightarrow R^{1}$ are summable functions and $h_{i j}^{k}:[0, \omega] \rightarrow[0, \omega]$ are measurable functions, $i, j=1, \ldots, n, k=1, \ldots, m$.

Corollary 2.3. Let the following conditions be fulfilled:

(1) at least one of the following assumptions (1a) or (1b) is satisfied:

(a) $h_{i i}^{k}(t) \leq t, t \in[0, \omega], i=1, \ldots, n, k=1, \ldots, m$, and the inequalities

$$
\begin{aligned}
& \sum_{k=1}^{m} p_{i i}^{k}(t) \geq 0, \quad t \in[0, \omega], \quad \sum_{k=1}^{m} p_{i i}^{k} \not \equiv 0, \quad i=1, \ldots, n, \\
& \int_{h_{i i}^{0}(t)}^{t} \sum_{k=1}^{m} p_{i i}^{k+}(s) d s \leq \frac{1}{e} \quad t \in[0, \omega], \quad i=1, \ldots, n,
\end{aligned}
$$

hold, where $h_{i i}^{0}(t)=\min _{k=1, \ldots, m} h_{i i}^{k}(t), t \in[0, \omega], i=1, \ldots, n$,

(b) the inequalities

$$
\sum_{k=1}^{m}\left\|p_{i i}^{k+}\right\|_{L}<1, \quad \sum_{k=1}^{m}\left\|p_{i i}^{k-}\right\|_{L}<1,
$$

and

$$
\frac{\sum_{k=1}^{m}\left\|p_{i i}^{k-}\right\|_{L}}{1-\sum_{k=1}^{m}\left\|p_{i i}^{k-}\right\|_{L}}<\sum_{k=1}^{m}\left\|p_{i i}^{k+}\right\|_{L}
$$

hold for $i=1, \ldots, n$,

where $p_{i i}^{k+}$ and $p_{i i}^{k-}$ denote the positive and negative parts, respectively, of the function $p_{i i^{\prime}}^{k}$

(2) $p_{i j}^{k}(t) \leq 0, t \in[0, \omega], i, j=1, \ldots, n, i \neq j, k=1, \ldots, m$,

(3) there exists a vector $z=\operatorname{col}\left(z_{1}, \ldots, z_{n}\right)$ with all positive components such that

$$
\sum_{k=1}^{m} \sum_{j=1}^{n} p_{i j}^{k}(t) z_{j} \geq 1, \quad t \in[0, \omega], \quad i=1, \ldots, n .
$$


Then boundary value problem (2.26), (1.4) is uniquely solvable for each right hand side $f=\operatorname{col}\left(f_{1}, \ldots, f_{n}\right)$ and all elements $G_{i j}(t, s), i, j=1, \ldots, n$, of its Green's matrix $G(t, s)$ are non-negative and satisfy relation (2.20).

Remark 2.5. If instead of assumption (3) in Corollary 2.3 we assume that there exists a constant matrix $Y=\left\{y_{i j}\right\}_{i, j=1}^{n}$ with non-negative components possessing the property (2.12) such that relations

$$
\sum_{k=1}^{m} \sum_{r=1}^{n} p_{i r}^{k}(t) y_{r j} \geq \delta_{i j}, \quad t \in[0, \omega], \quad i, j=1, \ldots, n,
$$

are fulfilled with $\delta_{i j}$ given by (2.15), then the elements $G_{i j}(t, s), i, j=1, \ldots, n$, of the Green's matrix $G(t, s)$ satisfy the inequalities (2.21).

Remark 2.6. In the case of constant coefficients $p_{i j}^{k}$, the vector $z=\operatorname{col}\left(z_{1}, \ldots, z_{n}\right)$ appearing in assumption (3) of Corollary $2.3 \mathrm{can}$ be found as a positive solution of the algebraic system

$$
\sum_{j=1}^{n} \sum_{k=1}^{m} p_{i j}^{k} z_{j}=1, \quad i=1, \ldots, n,
$$

which then guarantees that

$$
\int_{0}^{\omega} \sum_{j=1}^{n} G_{i j}(t, s) d s=z_{i}, \quad t \in[0, \omega], \quad i=1, \ldots, n .
$$

Analogously we can get the equality

$$
\int_{0}^{\omega} G_{i j}(t, s) d s=y_{i j}, \quad t \in[0, \omega], \quad i, j=1, \ldots, n,
$$

in Corollary 2.3 if $\left\{y_{i j}\right\}_{i, j=1}^{n}$ is a non-negative solution of the matrix equation

$$
\sum_{r=1}^{n} \sum_{k=1}^{m} p_{i r}^{k} y_{i j}=\delta_{i j}, \quad i, j=1, \ldots, n,
$$

with $\delta_{i j}$ given by relation (2.15) possessing the property (2.12).

\section{Positivity of Green's matrices of systems of FDEs-main results}

In this section, along with problem (1.1), (1.4) we consider the following auxiliary problem consisting of the $n$-1-dimensional system

$$
\left(m_{i} x\right)(t) \equiv x_{i}^{\prime}(t)+\sum_{j=1}^{n-1}\left(B_{i j} x_{j}\right)(t)=f_{i}(t), \quad t \in[0, \omega], \quad i=1, \ldots, n-1,
$$

and the boundary conditions

$$
x_{i}(0)=x_{i}(\omega), \quad i=1, \ldots, n-1 .
$$

Assuming that problem (3.1), (3.2) is uniquely solvable for each $\operatorname{col}\left(f_{1}, \ldots, f_{n-1}\right)$, we denote by $K(t, s)=\left\{K_{i j}(t, s)\right\}_{i, j=1}^{n-1}$ its Green's matrix. As above we denote (if exists) by $G(t, s)=\left\{G_{i j}(t, s)\right\}_{i, j=1}^{n}$ Green's matrix of periodic problem (1.1), (1.4). 
Theorem 3.1. Let the following conditions be fulfilled:

(1) Green's functions $g_{i}(t, s), i=1, \ldots, n-1$, of $n-1$ scalar periodic problems (2.1), (2.2) exist, are non-negative, and satisfy inequality (2.4) for each positive measurable essentially bounded function $\phi$,

(2) all non-diagonal operators $B_{i j}, i, j=1, \ldots, n-1, i \neq j$, are negative,

(3) there exists a vector $z=\operatorname{col}\left(z_{1}, \ldots, z_{n-1}\right)$ with all positive components such that

$$
\sum_{j=1}^{n-1}\left(B_{i j} z_{j}\right)(t) \geq 1, \quad t \in[0, \omega], \quad i=1, \ldots, n-1 .
$$

Then periodic problem (3.1), (3.2) is uniquely solvable for each $\operatorname{col}\left(f_{1}, \ldots, f_{n-1}\right)$, all elements $K_{i j}(t, s), i, j=1, \ldots, n-1$, of its Green's matrix $K(t, s)$ are non-negative,

$$
\int_{0}^{\omega} \sum_{j=1}^{n-1} K_{i j}(t, s) d s \leq z_{i}, \quad t \in[0, \omega], \quad i=1, \ldots, n-1,
$$

and the following assertions are true:

(a) if Green's function $g(t, s)$ of the scalar periodic problem

$$
\begin{aligned}
& x^{\prime}(t)+(B x)(t)=f^{*}(t), \quad t \in[0, \omega], \\
& x(0)=x(\omega),
\end{aligned}
$$

where the operator $B: C_{[0, \omega]} \rightarrow L_{[0, \omega]}$ is defined by the equality

$$
(B y)(t) \equiv-\sum_{i=1}^{n-1}\left(B_{n i}\left\{\int_{0}^{\omega} \sum_{j=1}^{n-1} K_{i j}(\cdot, s)\left(B_{j n} y\right)(s) d s\right\}\right)(t)+\left(B_{n n} \gamma\right)(t)
$$

and $f^{*} \in L_{[0, \omega]}$, exists and is positive, then periodic problem (1.1), (1.4) is uniquely solvable for each right hand side $f=\operatorname{col}\left(f_{1}, \ldots, f_{n}\right)$ and the elements $G_{n j}(t, s), j=1, \ldots, n$, of its Green's matrix $G(t, s)$ satisfy the relations

$$
G_{n j}(t, s) \geq 0, \quad t, s \in[0, \omega], \quad j=1, \ldots, n,
$$

in the case of negative operators $B_{n i} i=1, \ldots, n-1$, and relations

$$
G_{n n}(t, s) \geq 0, \quad G_{n j}(t, s) \leq 0, \quad t, s \in[0, \omega], \quad j=1, \ldots, n-1,
$$

in the case of positive operators $B_{n i} i=1, \ldots, n-1$,

(b) if Green's function $g(t, s)$ of the scalar periodic problem (3.5), (3.6), where the operator $B: C_{[0, \omega]} \rightarrow L_{[0, \omega]}$ is defined by equality (3.7) and $f^{\prime \prime} \in L_{[0, \omega]}$, exists and is negative, then periodic problem (1.1), (1.4) is uniquely solvable for each right hand side $f=\operatorname{col}\left(f_{1}, \ldots, f_{n}\right)$ and the elements $G_{n j}(t, s), j=1, \ldots, n$, of its Green's matrix $G(t, s)$ satisfy the relations

$$
G_{n j}(t, s) \leq 0, \quad t, s \in[0, \omega], \quad j=1, \ldots, n,
$$

in the case of negative operators $B_{n i} i=1, \ldots, n-1$, and relations

$$
G_{n n}(t, s) \leq 0, \quad G_{n j}(t, s) \geq 0, \quad t, s \in[0, \omega], \quad j=1, \ldots, n-1,
$$

in the case of positive operators $B_{n i} i=1, \ldots, n-1$. 
Remark 3.1. If instead of assumption (3) in Theorem 3.1 we assume that there exists a constant matrix $Y=\left\{y_{i j}\right\}_{i, j=1}^{n-1}$ with non-negative components possessing the property

$$
\sum_{j=1}^{n-1} y_{i j}>0, \quad i=1, \ldots, n-1,
$$

such that relations

$$
\sum_{k=1}^{n-1}\left(B_{i k} y_{k j}\right)(t) \geq \delta_{i j}, \quad t \in[0, \omega], \quad i, j=1, \ldots, n-1,
$$

are fulfilled with $\delta_{i j}$ given by (2.15), then the elements $K_{i j}(t, s), i, j=1, \ldots, n-1$, of the Green's matrix $K(t, s)$ satisfy the inequalities

$$
\int_{0}^{\omega} K_{i j}(t, s) d s \leq y_{i j,} \quad t \in[0, \omega], \quad i, j=1, \ldots, n-1 .
$$

Theorem 3.2. Let the following conditions be fulfilled:

(1) Green's functions $g_{i}(t, s), i=1, \ldots, n-1$, of $n-1$ scalar periodic problems (2.1), (2.2) exist, are non-positive, and satisfy inequality (2.4) for each positive measurable essentially bounded function $\phi$,

(2) all non-diagonal operators $B_{i j}, i, j=1, \ldots, n-1, i \neq j$, are positive,

(3) there exists a vector $z=\operatorname{col}\left(z_{1}, \ldots, z_{n-1}\right)$ with all positive components such that

$$
\sum_{j=1}^{n-1}\left(B_{i j} z_{j}\right)(t) \leq-1, \quad t \in[0, \omega], \quad i=1, \ldots, n-1 .
$$

Then periodic problem (3.1), (3.2) is uniquely solvable for each $\operatorname{col}\left(f_{1}, \ldots, f_{n-1}\right)$, all elements $K_{i j}(t, s), i, j=1, \ldots, n-1$, of its Green's matrix $K(t, s)$ are non-positive,

$$
-\int_{0}^{\omega} \sum_{j=1}^{n-1} K_{i j}(t, s) d s \leq z_{i} \quad t \in[0, \omega], \quad i=1, \ldots, n-1,
$$

and the following assertions are true:

(a) if Green's function $g(t, s)$ of the scalar periodic problem (3.5), (3.6), where the operator $B: C_{[0, \omega]} \rightarrow L_{[0, \omega]}$ is defined by equality (3.7) and $f^{*} \in L_{[0, \omega]}$, exists and is positive, then periodic problem (1.1), (1.4) is uniquely solvable for each right hand side $f=$ $\operatorname{col}\left(f_{1}, \ldots, f_{n}\right)$ and the elements $G_{n j}(t, s), j=1, \ldots, n$, of its Green's matrix $G(t, s)$ satisfy the relations (3.8) in the case of positive operators $B_{n \dot{v}} i=1, \ldots, n-1$, and relations (3.9) in the case of negative operators $B_{n i} i=1, \ldots, n-1$,

(b) if Green's function $g(t, s)$ of the scalar periodic problem (3.5), (3.6), where the operator $B: C_{[0, \omega]} \rightarrow L_{[0, \omega]}$ is defined by equality (3.7) and $f^{*} \in L_{[0, \omega]}$, exists and is negative, then periodic problem (1.1), (1.4) is uniquely solvable for each right hand side $f=\operatorname{col}\left(f_{1}, \ldots, f_{n}\right)$ and the elements $G_{n j}(t, s), j=1, \ldots, n$, of its Green's matrix $G(t, s)$ satisfy the relations (3.10) in the case of positive operators $B_{n i v} i=1, \ldots, n-1$, and relations (3.11) in the case of negative operators $B_{n i} i=1, \ldots, n-1$.

Remark 3.2. If instead of assumption (3) in Theorem 3.2 we assume that there exists a constant matrix $Y=\left\{y_{i j}\right\}_{i, j=1}^{n-1}$ with non-negative components possessing the property 
(3.12) such that relations

$$
\sum_{k=1}^{n-1}\left(B_{i k} \gamma_{k j}\right)(t) \leq-\delta_{i j}, \quad t \in[0, \omega], i, j=1, \ldots, n-1,
$$

are fulfilled with $\delta_{i j}$ given by (2.15), then the elements $K_{i j}(t, s), i, j=1, \ldots, n-1$, of the Green's matrix $K(t, s)$ satisfy the inequalities

$$
-\int_{0}^{\omega} K_{i j}(t, s) d s \leq y_{i j}, \quad t \in[0, \omega], \quad i, j=1, \ldots, n-1 .
$$

Corollary 3.1. Let the following conditions be fulfilled:

(1) the operators $B_{i i}, i=1, \ldots, n-1$, admit the representation $B_{i i}=B_{i i}^{+}-B_{i i}^{-}$, where$B_{i i}^{+} B_{i i}^{-}: C_{[0, \omega]} \rightarrow L_{[0, \omega]}$ are linear positive operators, and at least one of the following assumptions (1a) or (1b) is satisfied:

(a) $B_{i i}^{+}, B_{i i}^{-}, i=1, \ldots, n-1$, are 0 -Volterra operators,

$$
\left(B_{i i} 1\right)(t) \geq 0, \quad t \in[0, \omega], \quad B_{i i} 1 \not \equiv 0, \quad i=1, \ldots, n-1
$$

and

$$
\int_{h_{i * *}^{+}(t)}^{t}\left(B_{i i}^{+} 1\right)(s) d s \leq \frac{1}{e}, \quad t \in[0, \omega], \quad i=1, \ldots, n-1,
$$

(b) the inequalities (2.18) and (2.19) hold for $i=1, \ldots, n-1$,

(2) all non-diagonal operators $B_{i j}, i, j=1, \ldots, n-1, i \neq j$, are negative,

(3) there exists a constant matrix $Y=\left\{\gamma_{i j}\right\}_{i, j=1}^{n-1}$ with non-negative components possessing the property (3.12) such that inequalities (3.13) are fulfilled, where $\delta_{i j}$ is given by relation (2.15),

(4) the operator $B_{i n}, i=1, \ldots, n$, admit the representation $B_{\text {in }}=B_{i n}^{+}-B_{\text {in }}^{-}$, where $B_{i n^{\prime}}^{+} B_{i n}^{-}: C_{[0, \omega]} \rightarrow L_{[0, \omega]}$ are linear positive operators, and moreover $B_{j n}^{+} 1, B_{j n}^{-} 1 \in L_{[o, \omega]}^{\infty}, j=1, \ldots, n-1$. ${ }^{\mathrm{a}}$

Then the following assertions are true:

(i) if $B_{n i} i=1, \ldots, n-1$, are negative operators and the inequalities

$$
A^{+}<1, \quad A^{-}<1, \quad \frac{A^{-}}{1-A^{-}}<\left\|B_{n n}^{+} 1\right\|_{L}
$$

are fulfilled, where

$$
A^{+}=\left\|B_{n n}^{+} 1+\sum_{i=1}^{n-1}\left|B_{n i} 1\right| \sum_{j=1}^{n-1} y_{i j}|| B_{j n}^{+} 1\right\|_{L^{\infty}} \|_{L}
$$

and

$$
A^{-}=\left\|B_{n n}^{-} 1+\sum_{i=1}^{n-1}\left|B_{n i} 1\right| \sum_{j=1}^{n-1} y_{i k}|| B_{j n}^{-} 1\right\|_{L^{\infty}} \|_{L},
$$


then periodic problem (1.1), (1.4) is uniquely solvable for each right hand side $f=\mathrm{col}$ $\left(f_{1}, \ldots, f_{n}\right)$ and the elements $G_{n j}(t, s), j=1, \ldots, n$, of its Green's matrix $G(t, s)$ satisfy the inequalities (3.8),

(ii) if $B_{n i} i=1, \ldots, n-1$, are negative operators and the inequalities

$$
A^{+}<1, \quad A^{-}<1, \quad \frac{A^{+}}{1-A^{+}}<\left\|B_{n n}^{-} 1\right\|_{L}
$$

are fulfilled, where the numbers $A^{+}$and $A^{-}$are given by the relations (3.19) and (3.20), respectively, then periodic problem (1.1), (1.4) is uniquely solvable for each right hand side $f=\operatorname{col}\left(f_{1}, \ldots, f_{n}\right)$ and the elements $G_{n j}(t, s), j=1, \ldots, n$, of its Green's matrix $G$ $(t, s)$ satisfy the inequalities (3.10),

(iii) if $B_{n i} i=1, \ldots, n-1$, are positive operators and the inequalities (3.18) are fulfilled, where

$$
A^{+}=\left\|B_{n n}^{+} 1+\sum_{i=1}^{n-1} B_{n i} 1 \sum_{j=1}^{n-1} y_{i j}\right\| B_{j n}^{-} 1\left\|_{L^{\infty}}\right\|_{L}
$$

and

$$
A^{-}=\left\|B_{n n}^{-} 1+\sum_{i=1}^{n-1} B_{n i} 1 \sum_{j=1}^{n-1} y_{i k}\right\| B_{j n}^{+} 1\left\|_{L^{\infty}}\right\|_{L},
$$

then periodic problem (1.1), (1.4) is uniquely solvable for each right hand side $f=\mathrm{col}$ $\left(f_{1}, \ldots, f_{n}\right)$ and the elements $G_{n j}(t, s), j=1, \ldots, n$, of its Green's matrix $G(t, s)$ satisfy the inequalities (3.9),

(iv) if $B_{n i} i=1, \ldots, n-1$, are positive operators and the inequalities (3.21) are fulfilled, where the numbers $A+$ and $A^{-}$are given by the relations (3.22) and (3.23), respectively, then periodic problem (1.1), (1.4) is uniquely solvable for each right hand side $f=$ $\operatorname{col}\left(f_{1}, \ldots, f_{n}\right)$ and the elements $G_{n j}(t, s), j=1, \ldots, n$, of its Green's matrix $G(t, s)$ satisfy the inequalities (3.11).

\section{Proofs}

The main results of the article are based on the following assertion, which explains how the scalar functional differential equation for the component $x_{n}$ of the solution vector can be constructed.

Lemma 4.1. Let there exist Green's matrix $K(t, s)=\left\{K_{i j}(t, s)\right\}_{i, j=1}^{n-1}$ of periodic problem (3.1), (3.2). Then $\operatorname{col}\left(x_{1}, \ldots, x_{n}\right)$ is a solution of periodic problem (1.1), (1.4) if and only if $x_{n}$ is a solution of scalar periodic problem (3.5), (3.6) and

$$
\begin{aligned}
x_{i}(t)= & -\int_{0}^{\omega} \sum_{j=1}^{n-1} K_{i j}(t, s)\left(B_{j n} x_{n}\right)(s) d s \\
& +\int_{0}^{\omega} \sum_{j=1}^{n-1} K_{i j}(t, s) f_{j}(s) d s, \quad t \in[0, \omega], \quad i=1, \ldots, n-1,
\end{aligned}
$$


where the operator $B$ is defined by the equality (3.7) and

$$
f^{*}(t)=f_{n}(t)-\sum_{i=1}^{n-1}\left(B_{n i}\left\{\int_{0}^{\omega} \sum_{j=1}^{n-1} K_{i j}(\cdot, s) f_{j}(s) d s\right\}\right)(t), \quad t \in[0, \omega] .
$$

Proof. If $\left(x_{1}, \ldots, x_{n}\right)$ is a solution of periodic problem (1.1), (1.4) then, using Green's matrix $K(t, s)=\left\{K_{i j}(t, s)\right\}_{i, j=1}^{n-1}$ of problem (3.1), (3.2), we obtain that the components $x_{i}$, $i=1, \ldots, n-1$, satisfy the relations (4.1). Substitution of these representations in the $n$th equation of the system (1.1) leads to the fact that $x_{n}$ is a solution of scalar periodic problem (3.5), (3.6) in which the operator $B$ and the function $f^{*}$ are defined by formulas (3.7) and (4.2), respectively.

The converse implication can be verified easily by using Green's matrix $K(t, s)$ of the periodic problem (3.1), (3.2).

Lemma 4.2. [16] Let the operator $B$ admit the representation $B=B^{+}-B^{-}$, where $B^{+}$, $B^{-}: C_{[0, \omega]}: L_{[0, \omega]}$ are linear positive operators. If

$$
\left\|B^{+} 1\right\|_{L}<1, \quad\left\|B^{-} 1\right\|_{L}<1,
$$

and

$$
\frac{\left\|B^{-} 1\right\|_{L}}{1-\left\|B^{-} 1\right\|_{L}}<\left\|B^{+} 1\right\|_{L}
$$

then problem (3.5), (3.6) is uniquely solvable for each $f^{\prime \prime} \in L_{[0, \omega]}$ and its Green's function $g(t, s)$ is positive.

Lemma 4.3. [16] Let the operator $B$ admit the representation $B=B^{+}-B^{-}$, where $B^{+}$, $B^{-}: C_{[0, \omega]} \rightarrow L_{[0, \omega]}$ are linear positive operators. If the inequalities (4.3) hold and

$$
\frac{\left\|B^{+} 1\right\|_{L}}{1-\left\|B^{+} 1\right\|_{L}}<\left\|B^{-} 1\right\|_{L}
$$

then problem (3.5), (3.6) is uniquely solvable for each $f^{\prime \prime} \in L_{[0, \omega]}$ and its Green's function $g(t, s)$ is negative.

Lemma 4.4. [13] Let $B$ be an operator admitting the representation $B=B+-B^{-}$, where $B^{+}, B^{-}: C_{[0, \omega]} \rightarrow L_{[0, \omega]}$ are linear, positive, and 0-Volterra operators. If

$$
(B 1)(t) \geq 0, \quad t \in[0, \omega], \quad B 1 \not \equiv 0
$$

and

$$
\int_{h_{*}^{+}(t)}^{t}\left(B^{+} 1\right)(s) d s \leq \frac{1}{e}, \quad t \in[0, \omega]
$$

then problem (3.5), (3.6) is uniquely solvable for each $f^{\prime \prime} \in L_{[0, \omega]}$ and its Green's function $g(t, s)$ is positive.

Analogously to Lemma 4.5 one can prove the following assertion:

Lemma 4.5. Let $B$ be an operator admitting the representation $B=B^{+}-B^{-}$, where $B^{+}$, $B^{-}: C_{[0, \omega]} \rightarrow L_{[0, \omega]}$ are linear, positive, and $\omega$-Volterra operators. If

$$
(B 1)(t) \leq 0, \quad t \in[0, \omega], \quad B 1 \not \equiv 0
$$


and

$$
\int_{t}^{h^{-*}(t)}\left(B^{-} 1\right)(s) d s \leq \frac{1}{e}, \quad t \in[0, \omega],
$$

then problem (3.5), (3.6) is uniquely solvable for each $f^{\prime \prime} \in L_{[0, \omega]}$ and its Green's function $g(t, s)$ is negative.

Now we are in a position to prove the statements formulated in Sections 2 and 3.

Proof of Corollary 2.1. Lemma 4.4 (respectively, Lemma 4.2) implies that condition (1a) (respectively, condition (1b)) is sufficient for the existence and positivity of Green's functions $g_{i}(t, s), i=1, \ldots, n$, of periodic problems (2.1), (2.2) and for the validity of relation (2.4).

Consequently, the assertion of the corollary follows from Theorem 2.2.

Proof of Corollary 2.2. It can be proven analogously as Corollary 2.1 on the basis of Lemmas 4.3 and 4.5 .

Proof of Corollary 2.3. It follows from Corollary 2.1 as a particular case of the operators $B_{i j}$.

Proof of Theorem 3.1. In view of the assumptions (1)-(3), Theorem 2.2 yields that Green's matrix $K(t, s)$ of the periodic problem for auxiliary system (3.1) exists and its elements $K_{i, j}(t, s), i, j=1, \ldots, n-1$, are non-negative and satisfy the inequalities (3.4). The assertions (a) and (b) follow from analysis of signs in formula (4.2).

Indeed, consider the case when Green's function $g(t, s)$ of the scalar periodic problem (3.5), (3.6) with the operator $B: C_{[0, \omega]} \rightarrow L_{[0, \omega]}$ defined by equality (3.7) and $f^{*} \in L$ $[0, \omega]$ exists and is positive. Then, according to Lemma 4.1, the periodic problem (1.1), (1.4) has a unique solution $\operatorname{col}\left(x_{1}, \ldots, x_{n}\right)$ for an arbitrary right hand side $f=\operatorname{col}\left(f_{1}, \ldots\right.$, $f_{n}$ ) whereas

$$
x_{n}(t)=\int_{0}^{\omega} g(t, s) f^{*}(s) d s, \quad t \in[0, \omega],
$$

and $x_{i}, i=1, \ldots, n-1$, satisfy equalities (4.1), where the function $f^{\prime \prime}$ is given by formula (4.2). It is clear that non-negativity of $K_{i j}(t, s), i, j=1, \ldots, n-1$, in the case of negativity of the operators $B_{n i}, i=1, \ldots, n-1$, implies that non-negative $f_{j}, j=1, \ldots, n$, give non-negative $f^{*}$. From the equalities (4.8) and

$$
x_{n}(t)=\int_{0}^{\omega} \sum_{j=1}^{n} G_{n j}(t, s) f_{j}(s) d s, \quad t \in[0, \omega],
$$

it follows desired relation (3.8). In the case of positivity of the operators $B_{n i}, i=1, \ldots$, $n-1$, the inequalities $f_{i} \leq 0, i=1, \ldots, n-1, f_{n} \geq 0$ give non-negative $f^{*}$. Consequently, equalities (4.8) and (4.9) lead to desired relation (3.9).

Consider now the case when Green's function $g(t, s)$ of the scalar periodic problem (3.5), (3.6) with the operator $B: C_{[0, \omega]} \rightarrow L_{[0, \omega]}$ defined by equality (3.7) and $f^{\prime \prime} \in L_{[0, \omega]}$ exists and is negative. One can show analogously as above that non-negativity of $K_{i j}(t$, $s), i, j=1, \ldots, n-1$, in the case of negativity of the operators $B_{n i}, i=1, \ldots, n-1$, implies relation (3.10) and in the case of positivity of the operators $B_{n i}, i=1, \ldots, n-1$, yields relation (3.11). 
Proof of Theorem 3.2. It can be proven analogously as Theorem 3.1.

Proof of Corollary 3.1. According to Lemmas 4.2 and 4.4, each of the condition (1a) and (1b) implies that Green's functions $g_{i}(t, s), i=1, \ldots, n-1$, of periodic problems (2.1), (2.2) exist, are positive, and satisfy relation (2.4). Therefore, in view of assumptions (2) and (3), Theorem 3.1 (see also Remark 3.1) guarantees that the elements $K_{i j}$, $i, j=1, \ldots, n-1$, of Green's matrix $K(t, s)$ of the periodic problem (3.1), (3.2) are positive and satisfy inequalities (3.14).

(i) Let us define the operator $B: C_{[0, \omega]} \rightarrow L_{[0, \omega]}$ by equality (3.7). Then, in view of assumption (4) and relations (3.14), the operator $B$ admits the representation $B=B^{+}$$B^{-}$, where $B^{+}, B^{-}: C_{[0, \omega]} \rightarrow L_{[0, \omega]}$ are linear positive operators such that

$$
\left\|B_{n n}^{+} 1\right\|_{L} \leq\left\|B^{+} 1\right\|_{L} \leq A^{+}, \quad\left\|B^{-} 1\right\|_{L} \leq A^{-} .
$$

Therefore, using Lemma 4.2, we get the existence and positivity of Green's function $g$ $(t, s)$ of the periodic problem for scalar equation (3.5), and thus the assertion follows from Theorem 3.1(a).

(ii) According to assumption (4) and relations (3.14), it is clear that the operator $B: C$ $[0, \omega] \rightarrow L_{[0, \omega]}$ defined by equality (3.7) admits the representation $B=B^{+}-B^{-}$, where $B^{+}$, $B^{-}: C_{[0, \omega]} \rightarrow L_{[0, \omega]}$ are linear positive operators such that

$$
\left\|B^{+} 1\right\|_{L} \leq A^{+}, \quad\left\|B_{n n}^{-} 1\right\|_{L} \leq\left\|B^{-} 1\right\|_{L} \leq A^{-} .
$$

Therefore, Lemma 4.3 yields that Green's matrix of periodic problem (3.5), (3.6) exists and is negative, and thus the assertion follows from Theorem 3.1(b).

Assertions (iii) and (iv) can be proven analogously.

\section{Two-dimensional systems with argument deviations}

Consider the periodic problem for the system

$$
\begin{aligned}
& x^{\prime}{ }_{1}(t)+p_{11}(t) x_{1}\left(h_{11}(t)\right)+p_{12}(t) x_{2}\left(h_{12}(t)\right)=f_{1}(t), \\
& x^{\prime}{ }_{2}(t)+p_{21}(t) x_{1}\left(h_{21}(t)\right)+p_{22}(t) x_{2}\left(h_{22}(t)\right)=f_{2}(t),
\end{aligned}, t \in[0, \omega],
$$

where $p_{i j}, f_{i}:[0, \omega] \rightarrow R^{1}$ are summable functions and the functions $h_{i j}:[0, \omega] \rightarrow$ $[0, \omega]$ are measurable, $i, j=1,2$.

Let us introduce the notation:

$$
P(t)=\left(\begin{array}{c}
+- \\
-+
\end{array}\right)
$$

means that $p_{11}(t) \geq 0, p_{12}(t) \leq 0, p_{21}(t) \leq 0, p_{22}(t) \geq 0, t \in[0, \omega]$. Moreover, having a measurable essentially bounded function $p:[0, \omega] \rightarrow[0, \omega]$, we set

$$
p_{*}=\operatorname{ess} \inf \{p(t): t \in[0, \omega]\}, \quad p^{*}=\operatorname{ess} \sup \{p(t): t \in[0, \omega]\} .
$$

Theorem 5.1. Let the coefficients $p_{i j} i, j=1,2$, be essentially bounded and the following conditions be fulfilled:

(1) $0<\int_{0}^{\omega}\left|p_{i i}(t)\right| d t<1$ for $i=1,2$,

(2) $\Delta:=\left|p_{11}\right| "\left|p_{22}\right| "-\left|p_{12}\right|^{*}\left|p_{21}\right|^{\prime \prime}>0$.

Then Green's matrix $G(t, s)$ of the periodic problem for system (5.1) exists and satisfies: 
(a)

$$
G(t, s)=\left(\begin{array}{c}
++ \\
++
\end{array}\right) \quad \text { if } \quad P(t)=\left(\begin{array}{c}
+- \\
-+
\end{array}\right),
$$

(b)

$$
G(t, s)=\left(\begin{array}{l}
-- \\
--
\end{array}\right) \quad \text { if } \quad P(t)=\left(\begin{array}{c}
-+ \\
+-
\end{array}\right),
$$

(c)

$$
G(t, s)=\left(\begin{array}{c}
++ \\
--
\end{array}\right) \quad \text { if } \quad P(t)=\left(\begin{array}{c}
+- \\
+-
\end{array}\right),
$$

(d)

$$
G(t, s)=\left(\begin{array}{c}
-- \\
++
\end{array}\right) \quad \text { if } \quad P(t)=\left(\begin{array}{c}
-+ \\
-+
\end{array}\right) .
$$

Proof. Let the operators $B_{i j}: C_{[0, \omega]} \rightarrow L_{[0, \omega]}$ be defined by the relations

$$
B_{i j}(z)(t)=p_{i j}(t) z\left(h_{i j}(t)\right), \quad t \in[0, \omega], \quad i, j=1,2 .
$$

Then system (5.1) is a particular case of system (1.1) with $n=2$.

According to assumption (2), the algebraic system

$$
\begin{aligned}
\left|p_{11}\right|_{*} z_{1}-\left|p_{12}\right|^{*} z_{2} & =1, \\
-\left|p_{21}\right|^{*} z_{1}+\left|p_{22}\right|_{*} z_{2} & =1
\end{aligned}
$$

has a solution $z_{1}=\left(\left|p_{22}\right| *+\left|p_{12}\right|^{*}\right) / \Delta>0$ and $z_{2}=\left(\left|p_{11}\right| *+\left|p_{21}\right|^{*}\right) / \Delta>0$. This solution

satisfies also the inequalities

$$
\begin{gathered}
\left|p_{11}(t)\right| z_{1}-\left|p_{12}(t)\right| z_{2} \geq 1, \\
-\left|p_{21}(t)\right| z_{1}+\left|p_{22}(t)\right| z_{2} \geq 1,
\end{gathered}
$$

$t \in[0, \omega]$, and thus the assertions of the theorem follows from Theorem 2.1 with $v$ $(t) \equiv \operatorname{col}\left(z_{1}, z_{2}\right)$ and Lemmas 4.2 and 4.3 .

Theorem 5.2. Let the coefficients $p_{i j}, i, j=1,2$, be essentially bounded and the following conditions hold:

(1) $0<\int_{0}^{\omega}\left|p_{11}(t)\right| d t<1$,

(2) $\left.\left|p_{12}\right|^{*} \int_{0}^{\omega} p_{21}(t)|d t<| p_{11}\right|_{*}$

(3) $\Delta:=\left|p_{11}\right|^{*}\left|p_{22}\right|^{*}-\left|p_{12}\right|_{*}\left|p_{21}\right|_{*}<0$.

Then Green's matrix $G(t, s)$ of the periodic problem for system (5.1) exists and satisfies:

(a)

$$
G(t, s)=\left(\begin{array}{c}
\cdot \cdot \\
--
\end{array}\right) \text { if } P(t)=\left(\begin{array}{c}
+- \\
-+
\end{array}\right),
$$


(b)

$$
G(t, s)=\left(\begin{array}{c}
\cdot \\
++
\end{array}\right) \text { if } P(t)=\left(\begin{array}{c}
-+ \\
+-
\end{array}\right),
$$

(c)

$$
G(t, s)=\left(\begin{array}{c}
\cdot \cdot \\
-+
\end{array}\right) \quad \text { if } \quad P(t)=\left(\begin{array}{c}
+- \\
+-
\end{array}\right)
$$

(d)

$$
G(t, s)=\left(\begin{array}{c}
\cdot \cdot \\
+-
\end{array}\right) \quad \text { if } \quad P(t)=\left(\begin{array}{c}
-+ \\
-+
\end{array}\right) .
$$

To prove Theorem 5.2 we need the following lemma.

Lemma 5.1. Let the operator $B$ admit the representation $B=B^{+}-B^{-}$, where $B^{+}, B^{-}: C$ $[0, \omega] \rightarrow L_{[0, \omega]}$ are linear positive operators, and let the Green's function of the problem

$$
y^{\prime}(t)-\left(B^{-} \gamma\right)(t)=f^{*}(t), \quad y(0)=y(\omega)
$$

exist and be negative. Let, moreover,

$$
(B 1)(t) \leq 0, \quad t \in[0, \omega], \quad B 1 \not \equiv 0 .
$$

Then the Green's function of the problem (3.5), (3.6) exists and is negative.

Proof. It is sufficient to show that any nontrivial absolutely continuous function $y:[0$, $\omega] \rightarrow R^{1}$ satisfying

$$
\gamma^{\prime}(t)+(B y)(t) \geq 0, \quad t \in[0, \omega], \quad \gamma(0)=\gamma(\omega)
$$

is negative. Obviously, if $y$ is non-positive, then from (5.5) it follows that

$$
\gamma^{\prime}(t)-\left(B^{-} \gamma\right)(t) \geq 0, \quad t \in[0, \omega], \quad \gamma(0)=\gamma(\omega),
$$

whence, according to the non-negativity of the Green's function of the problem (5.3), we get $y(t)<0$ for $t[0, \omega]$. Assume therefore that

$$
M=\max \{\gamma(t): t \in[0, \omega]\}>0
$$

and put

$$
w(t)=M-\gamma(t), \quad t \in[0, \omega] .
$$

Then $w(t) \geq 0$ for $t \in[0, \omega]$ and there exist $t_{0} \in[0, \omega]$ such that

$$
w\left(t_{0}\right)=0
$$

Furthermore, in view of (5.4) and (5.5) we have

$$
w^{\prime}(t)-\left(B^{-} w\right)(t) \leq w^{\prime}(t)+(B w)(t) \leq 0, \quad t \in[0, \omega] .
$$

Consequently, since the Green's function of the problem (5.3) is negative, on account of (5.6) we obtain $w \equiv 0$, i.e., $y(t)$ is a constant function. Now from (5.5) we obtain $(B 1)$ $(t) \geq 0$ for $t \in[0, \omega]$, which contradicts (5.4).

Proof of Theorem 5.2. Let the operators $B_{i j}: C_{[0, \omega]} \rightarrow L_{[0, \omega]}$ be defined by relations (5.2). Then system (5.1) is a particular case of system (1.1) with $n=2$. 
We will show only the assertion (a). Let the operator $B: C_{[0, \omega]} \rightarrow L_{[0, \omega]}$ be defined by formula (3.7) with $n=2$ in which $K_{11}(t, s)$ denotes Green's function of the scalar periodic problem

$$
x_{1}^{\prime}(t)+p_{11}(t) x_{1}\left(h_{11}(t)\right)=f_{1}(t), \quad t \in[0, \omega], \quad x_{1}(0)=x_{1}(\omega),
$$

the existence and positivity of which follows from Lemma 4.2 and assumption (1). Then we have

$$
\int_{0}^{\omega} K_{11}(t, s) d s \leq \frac{1}{\left|p_{11}\right|_{*}}, \quad t \in[0, \omega],
$$

and $B=B_{22}-A$, where the operator $A: C_{[0, \omega]} \rightarrow L_{[0, \omega]}$ is defined by the equality

$$
(A y)(t) \equiv p_{21}(t) \int_{0}^{\omega} K_{11}\left(h_{21}(t), s\right) p_{12}(s) y\left(h_{12}(s)\right) d s, \quad t \in[0, \omega] .
$$

The operator $A$ is positive and non-zero and thus, according to assumption (2) and relation (5.8), Lemma 4.3 guarantees that Green's function of the problem

$$
\gamma^{\prime}(t)-(A y)(t)=f^{*}(t), \quad y(0)=\gamma(\omega),
$$

exists and is negative. Therefore, it follows from Lemma 5.1 that Green's function $g$ $(t, s)$ of the problem (3.5), (3.6) exists and is negative if there exists a positive constant $z_{2}$ and $\varepsilon>0$ such that

$$
\left(B_{22} z_{2}\right)(t)-\left(A z_{2}\right)(t) \leq-\epsilon, \quad t \in[0, \omega] .
$$

Let us prove that such constants $z_{2}$ and $\varepsilon$ exist. According to assumption (3), the algebraic system

$$
\begin{aligned}
\left|p_{11}\right|^{*} z_{1}-\left|p_{12}\right|_{*} z_{2} & =-1, \\
-\left|p_{21}\right|_{*} z_{1}+\left|p_{22}\right|^{*} z_{2} & =-1
\end{aligned}
$$

has a solution $z_{1}=\left(-\left|p_{22}\right|^{*}-\left|p_{12}\right|^{*}\right) / \Delta>0$ and $z_{2}=\left(-\left|p_{11}\right|^{*}-\left|p_{21}\right|^{*}\right) / \Delta>0$. This solution satisfies also the inequalities

$$
\begin{aligned}
& p_{11}(t) z_{1}+p_{12}(t) z_{2}=\left|p_{11}(t)\right| z_{1}-\left|p_{12}(t)\right| z_{2} \leq-1, \\
& p_{21}(t) z_{1}+p_{22}(t) z_{2}=-\left|p_{21}(t)\right| z_{1}+\left|p_{22}(t)\right| z_{2} \leq-1,
\end{aligned}
$$

$t \in[0 . \omega]$, Therefore, $\operatorname{col}\left(z_{1}, z_{2}\right)$ is a solution of the periodic problem for system (5.1) with $f_{1}(t) \equiv\left|p_{11}(t)\right| z_{1}-\left|p_{12}(t)\right| z_{2}$ and $f_{2}(t) \equiv-\left|p_{21}(t)\right| z_{1}+\left|p_{22}(t)\right| z_{2}$, and Lemma 4.1 with $n=2$ then yields that

$$
\left(B z_{2}\right)(t)=f_{2}(t)-p_{21}(t) \int_{0}^{\omega} K_{11}\left(h_{21}(t), s\right) f_{1}(s) d s \leq-1, \quad t \in[0, \omega] .
$$

Consequently, relation (5.9) is satisfied with $\varepsilon=1$ and thus Green's function $g(t, s)$ of the problem (3.5), (3.6) exists and is negative.

Now it follows from Lemma 4.1 with $n=2$ that the periodic problem for system (5.1) has a unique solution $\operatorname{col}\left(x_{1}, x_{2}\right)$ for an arbitrary right hand side $\operatorname{col}\left(f_{1}, f_{2}\right)$ whereas 


$$
x_{2}(t)=\int_{0}^{\omega} g(t, s)\left(f_{2}(s)-p_{21}(s) \int_{0}^{\omega} K_{11}\left(h_{21}(s), \xi\right) f_{1}(\xi) d \xi\right) d s, \quad t \in[0, \omega] .
$$

It is clear that positivity of $K_{11}$ and negativity of $g$ imply that non-negative $f_{1}, f_{2}$ give non-positive $x_{2}$ and thus the representation

$$
x_{2}(t)=\int_{0}^{\omega}\left(G_{21}(t, s) f_{1}(s)+G_{22}(t, s) f_{2}(s)\right) d s, \quad t \in[0, \omega],
$$

guarantees the desired relations $G_{21}(t, s) \leq 0$ and $G_{22}(t, s)=0, t, s \in[0, \omega]$.

Example 5.1. Let us demonstrate that in very natural cases only elements in corresponding rows of Green's matrix and not all its elements can preserve their signs. Consider the periodic problem for a second order scalar differential equation

$$
\begin{aligned}
& y^{\prime \prime}(t)+p_{11}(t) \gamma^{\prime}\left(h_{11}(t)\right)+p_{12}(t) y\left(h_{12}(t)\right)=f_{1}(t), \quad t \in[0, \omega], \\
& x(0)=x(\omega), \quad x^{\prime}(0)=x^{\prime}(\omega),
\end{aligned}
$$

and the corresponding differential system

$$
\begin{aligned}
& x^{\prime}{ }_{1}(t)+p_{11}(t) x_{1}\left(h_{11}(t)\right)+p_{12}(t) x_{2}\left(h_{12}(t)\right)=f_{1}(t), \\
& x_{2}^{\prime}(t)-x_{1}(t)=0,
\end{aligned}
$$

with the periodic conditions

$$
x_{i}(0)=x_{i}(\omega), \quad i=1,2 .
$$

For problem (5.12), (5.13) the following assertion follows from Theorem 5.2.

Corollary 5.1. Let $p_{11}$ and $p_{12}$ be essentially bounded and let the following conditions hold:

(1) $0<\int_{0}^{\omega} p_{11}(s) d s<1$,

(2) $\left|p_{12}\right|^{*} \omega<p_{11^{*}}$

(3) $p_{12}^{*}<0$.

Then elements $G_{21}(t, s)$ and $G_{22}(t, s)$ of Green's matrix of the problem (5.12), (5.13) are non-positive for $t, s \in[0, \omega]$.

The element $G_{21}(t, s)$ of Green's matrix of the problem (5.12), (5.13) coincides with Green's function $W(t, s)$ of the periodic problem for the scalar second order equation (5.10), and $G_{11}(t, s)$ corresponds to $W_{t}^{\prime}(t, s)$. It is clear that for any $f_{1}$, the derivative $x^{\prime}$ of a non-constant solution to the problem (5.10), (5.11) changes its sign, and thus the element $G_{11}(t, s)$ also changes its sign.

Consider the system with constant coefficients

$$
\begin{aligned}
& x^{\prime}{ }_{1}(t)+p_{11} x_{1}\left(h_{11}(t)\right)+p_{12} x_{2}\left(h_{12}(t)\right)=f_{1}(t), \\
& x^{\prime}{ }_{2}(t)+p_{21} x_{1}\left(h_{21}(t)\right)+p_{22} x_{2}\left(h_{22}(t)\right)=f_{2}(t),
\end{aligned}, \quad t \in[0, \omega]
$$

where $p_{i j} \in R^{1}, f_{i}:[0, \omega] \rightarrow R^{1}$ are summable functions and $h_{i j}:[0, \omega] \rightarrow[0, \omega]$ are measurable, $i, j=1,2$.

Corollary 5.2. Let $p_{i j}, i, j=1,2$, be such that 
(1)

$$
P=\left(\begin{array}{c}
+- \\
-+
\end{array}\right)
$$

(2) $0<p_{i i} \omega<1, i=1,2$,

(3) $p_{12} p_{21} \omega<\min \left\{p_{11}, p_{22}\right\}$.

If $p_{11} p_{22}-p_{21} p_{12}>0$, then Green's matrix $G(t, s)$ of the periodic problem for system (5.14) exists and satisfies

$$
G(t, s)=\left(\begin{array}{c}
++ \\
++
\end{array}\right)
$$

If $p_{11} p_{22}-p_{21} p_{12}<0$, then Green's matrix $G(t, s)$ of the periodic problem for system (5.14) exists and satisfies

$$
G(t, s)=\left(\begin{array}{l}
-- \\
--
\end{array}\right) .
$$

Proof. It follows from Theorems 5.1 and 5.2.

Theorem 5.3. Let the coefficients $p_{11}$ and $p_{12}$ be essentially bounded and the following conditions hold:

(1) $0<\int_{0}^{\omega}\left|p_{11}(t)\right| d t<1$,

(2) $\int_{0}^{\omega} p_{22}(t) d t \neq 0$,

(3) $\left|p_{12}\right|^{*} \int_{0}^{\omega}\left|p_{21}(t)\right| d t<\left|p_{11}\right|_{*}\left(1-\int_{0}^{\omega}\left|p_{22}(t)\right| d t\right)$.

Then Green's matrix $G(t, s)$ of the periodic problem for system (5.1) exists and satisfies:

(a)

$$
G(t, s)=\left(\begin{array}{c}
\cdot \cdot \\
++
\end{array}\right) \quad \text { if } \quad P(t)=\left(\begin{array}{c}
-+ \\
++
\end{array}\right),
$$

(b)

$$
G(t, s)=\left(\begin{array}{c}
\cdot \cdot \\
++
\end{array}\right) \quad \text { if } P(t)=\left(\begin{array}{c}
++ \\
+-
\end{array}\right),
$$

(c)

$$
G(t, s)=\left(\begin{array}{c}
\cdot \cdot \\
-+
\end{array}\right) \quad \text { if } \quad P(t)=\left(\begin{array}{c}
+- \\
++
\end{array}\right),
$$

(d)

$$
G(t, s)=\left(\begin{array}{c}
\cdot \cdot \\
--
\end{array}\right) \quad \text { if } \quad P(t)=\left(\begin{array}{c}
+- \\
--
\end{array}\right) .
$$

Proof. Let the operators $B_{i j}: C_{[0, \omega]} \rightarrow L_{[0, \omega]}$ be defined by relations (5.2). Then system (5.1) is a particular case of system (1.1) with $n=2$.

We will show only the assertion (b). It is clear that condition (1) yields the validity of the assumption (1b) of Corollary 3.1. Moreover, we have

$$
p_{11}(t) \frac{1}{\left|p_{11}\right|_{*}} \geq 1, \quad t \in[0, \omega],
$$


and thus the assumption (3) of Corollary 3.1 is satisfied with $Y=y_{11}=1 /\left|p_{11}\right| *$. If we define numbers $A^{+}$and $A^{-}$by formulas (3.22) and (3.23), respectively, we obtain

$$
A^{+}=0, \quad A^{-}=\left\|\left|p_{22}\right|+p_{21} \frac{1}{\left|p_{11}\right|_{*}}\left|p_{12}\right|^{*}\right\|_{L} .
$$

Therefore, inequalities (3.21) are fulfilled, because $\left\|B_{n n}^{-} 1\right\|_{L}=\int_{0}^{\omega}\left|p_{22}(t)\right| d t$ in this case. Consequently, the assertion follows from Corollary 3.1(iv).

\section{Endnote}

${ }^{a}$ As usual, $L_{[0, \omega]}^{\infty}$ denotes the space of measurable and essentially bounded functions $y$ : $[0, \omega] \rightarrow R^{1}$ endowed with the norm $\|y\|_{L^{\infty}}=\operatorname{ess} \sup \{|\gamma(t)|: t \in[0, \omega]\}$.

\section{Acknowledgements}

Robert Hakl and Jiř Šremr were supported by RVO: 67985840

\section{Author details}

${ }^{1}$ Department of Mathematics and Computer Science, The Ariel University Center of Samaria, 44837 Ariel Israel ${ }^{2}$ Institute of Mathematics, Academy of Sciences of the Czech Republic, Branch in Brno, Žižkova 22, 61662 Brno, Czech Republic

\section{Authors' contributions}

$A D, R H$, and JS obtained the results in a joint research. All authors read and approved the final manuscript.

\section{Competing interests}

The authors declare that they have no competing interests.

Received: 1 February 2012 Accepted: 22 May 2012 Published: 22 May 2012

\section{References}

1. Azbelev, NV, Maksimov, VP, Rakhmatullina, LF: Introduction to the theory of functional differential equations. In Adv Ser Math Sci Eng, vol. 3,World Federation Publisher Company, Atlanta (1995)

2. Hakl, R, Mukhigulashvili, S: On a boundary value problem for $n$-th order linear functional differential systems. Georgian Math J. 12(2), 229-236 (2005)

3. Kiguradze, I, Půža, B: Boundary value problems for systems of linear functional differential equations. In Folia Facul Sci Natur Univ Masar Brun, Mathematica, vol. 12,Masaryk University, Brno (2003)

4. Tchaplygin, SA: New Method of Approximate Integration of Differential Equations. GTTl, Moscow-Leningrad (1932)

5. Luzin: About method of approximate integration of acad. S A Tchaplygin Uspekhi Mat Nauk 6(6), 3-27 (1951). (in Russian)

6. Lakshmikantham, V, Leela, S: Differential and integral inequalities. Academic Press, New York/London (1969)

7. Krasnosel'skii, MA, Vainikko, GM, Zabreiko, PP, Rutitskii, JaB, Stezenko, VJa: Approximate methods for solving operator equations.

8. Kiguradze, I, Půža, B: On boundary value problems for systems of linear functional differential equations. Czechoslovak Math J. 47(2), 341-373 (1997). doi:10.1023/A:1022829931363

9. Kiguradze, I: Boundary value problems for systems of ordinary differential equations. J Sov Math. 43, 2 (1988)

10. Ważewski, T: Systemes des equations et des inegalites differentielled aux deuxieme membres et leurs applications. Ann Soc Polon Math. 23, 112-166 (1950)

11. Agarwal, R, Domoshnitsky, A: On positivity of several components of solution vector for systems of linear functional differential equations. Glasgow Math J. 52, 115-136 (2010). doi:10.1017/S0017089509990218

12. Domoshnitsky, A: Differential inequalities for one component of solution vector for systems of linear functional differential equations. Adv Diff Equ 2010, 14 (2010). (Article ID 478020), doi:10.1155/2010/478020

13. Domoshnitsky, A: Maximum principles and nonoscillation intervals for first order Volterra functional differential equations. Dyn Contin Discr Impuls Syst Ser A Math Anal. 15, 769-814 (2008)

14. Hakl, R, Lomtatidze, A, Šremr, J: Some Boundary Value Problems for First Order Scalar Functional Differential Equations. In Facul Sci Natur Univ Masar Brun, Mathematica, vol. 10,Masaryk University, Brno (2002)

15. Berman, A, Plemmons, JR: Nonnegative Matrices in the Mathematical Sciences. Academic Press, New York/London (1979)

16. Hakl, R, Lomtatidze, A, Šremr, J: On a boundary-value problem of periodic type for first-order linear functional differential equations. Nonlinear Oscil. 5(3), 408-425 (2002). doi:10.1023/A:1022304626385 